\title{
Analysis of Organochlorine Pesticide Residues in Various Vegetable Oils Collected in Chinese Markets
}

Yang Cui ${ }^{1,4}$, Runhui $\mathrm{Ke}^{2, *}$, Wei Gao ${ }^{1}$, Feifei $\operatorname{Tian}^{5}$,Yawei Wang ${ }^{1,3,4, *}$, Guibin Jiang ${ }^{1}$

${ }^{1}$ Research Center for Eco-Environmental Sciences, Chinese Academy of Sciences, Beijing 100085, China

${ }^{2}$ Chinese National Research Institute of Food \& Fermentation Industries Co., Ltd, Beijing 100016, China

${ }^{3}$ School of Environment, Hangzhou Institute for Advanced Study, UCAS, Hangzhou 310000, China

${ }^{4}$ Sino-Danish College, University of Chinese Academy of Sciences, Beijing 100049, China

${ }^{5}$ Shimadzu Global COE for Application \& Technical Development, Beijing 100020, China

*Corresponding author

Dr. Yawei Wang

State Key Laboratory of Environmental Chemistry and Ecotoxicology

Research Center for Eco-Environmental Sciences

Chinese Academy of Sciences

P.O. Box 2871, Beijing 100085, China

Tel: +8610-6284-0620

Fax: $+8610-6284-9339$

E-mail: ywwang@,rcees.ac.cn 


\section{CONTENTS}

Table S1. The MDLs and recoveries of 26 kinds of pesticides.

Table S2. The detection rates for each pesticide of different vegetable oil types. ......S2

Table S3. Spearman's Rank Correlation Coefficient between Concentrations of OCPs in vegetable oils.

Table S4. DI for the pesticides in different vegetable oils for urban and rural households (ng/kg bw/day). S4

Table S5. HQ for the pesticides in different vegetable oils for urban and rural households S5 
Table S1. The MDLs and recoveries of 26 kinds of pesticides

\begin{tabular}{|c|c|c|c|c|c|}
\hline & MDLs/ng/g & recoveries & & MDLs/ng/g & recoveries \\
\hline Aldrin & 0.45 & $65-144 \%$ & trans-Chlordane & 0.14 & $70-103 \%$ \\
\hline Dieldrin & 0.15 & $72-135 \%$ & Pentachlorobenzene & 1.84 & $68-142 \%$ \\
\hline Mirex & 0.10 & $60-133 \%$ & Hexachlorobenzene & 2.77 & $59-148 \%$ \\
\hline Isodrin & 0.75 & $52-154 \%$ & o,p'-DDD & 0.21 & $59-154 \%$ \\
\hline Endrin & 0.25 & $75-121 \%$ & p,p'-DDD & 0.12 & $61-147 \%$ \\
\hline Endrin Aldehyde & 0.51 & $74-97 \%$ & o,p'-DDE & 0.10 & $49-150 \%$ \\
\hline Endrin Ketone & 0.10 & $70-101 \%$ & $\mathrm{p}, \mathrm{p}^{\prime}-\mathrm{DDE}$ & 0.13 & $76-159 \%$ \\
\hline Chlordene & 0.71 & $52-136 \%$ & o,p'-DDT & 0.15 & $85-149 \%$ \\
\hline $\begin{array}{c}1- \\
\text { Hydroxychlordene }\end{array}$ & 0.45 & $65-144 \%$ & $\mathrm{p}, \mathrm{p}^{\prime}-\mathrm{DDT}$ & 0.13 & $67-145 \%$ \\
\hline Heptachlor & 0.23 & $76-130 \%$ & $\begin{array}{l}\alpha \text {-Hexachloro } \\
\text { cyclohexane }\end{array}$ & 0.21 & $51-124 \%$ \\
\hline o,p'-Methoxychlor & 0.11 & $67-145 \%$ & $\begin{array}{l}\beta \text {-Hexachloro } \\
\text { cyclohexane }\end{array}$ & 0.55 & $61-123 \%$ \\
\hline p,p'-Methoxychlor & 0.12 & $70-101 \%$ & $\begin{array}{l}\gamma \text {-Hexachloro } \\
\text { cyclohexane }\end{array}$ & 0.35 & $44-128 \%$ \\
\hline cis-Chlordane & 0.97 & $69-137 \%$ & $\begin{array}{l}\delta \text {-Hexachloro } \\
\text { cyclohexane }\end{array}$ & 0.89 & $59-95 \%$ \\
\hline
\end{tabular}


Table S2. The detection rates for each pesticide of different vegetable oil types

\begin{tabular}{|c|c|c|c|c|c|c|c|c|c|c|c|}
\hline & $\mathrm{S}$ & $\mathrm{O}$ & $\mathrm{C}$ & Co & $\mathrm{Ca}$ & $\mathrm{P}$ & So & $\mathrm{L}$ & $\mathrm{B}$ & $\mathrm{Su}$ & $\mathrm{R}$ \\
\hline Total amount & 19 & 17 & 13 & 13 & 9 & 20 & 7 & 5 & 8 & 6 & 7 \\
\hline Aldrin & 19 & 17 & 13 & 13 & 9 & 20 & 6 & 5 & 8 & 6 & 7 \\
\hline Dieldrin & 19 & 15 & 9 & 11 & 7 & 13 & 6 & 4 & 4 & 4 & 3 \\
\hline Mirex & 19 & 17 & 13 & 13 & 9 & 20 & 7 & 5 & 8 & 5 & 7 \\
\hline Isodrin & 18 & 16 & 11 & 12 & 9 & 16 & 6 & 5 & 8 & 5 & 7 \\
\hline Endrin & 19 & 17 & 13 & 13 & 9 & 20 & 7 & 5 & 8 & 6 & 7 \\
\hline Endrin Aldehyde & 19 & 15 & 13 & 13 & 8 & 19 & 6 & 5 & 8 & 6 & 7 \\
\hline Endrin Ketone & 19 & 17 & 11 & 13 & 8 & 18 & 7 & 5 & 7 & 5 & 5 \\
\hline Chlordene & 19 & 17 & 13 & 13 & 9 & 20 & 7 & 5 & 8 & 6 & 7 \\
\hline 1-Hydroxychlordene & 10 & 14 & 10 & 11 & 7 & 13 & 4 & 4 & 6 & 5 & 6 \\
\hline Heptachlor & 17 & 17 & 12 & 11 & 7 & 17 & 5 & 5 & 7 & 6 & 6 \\
\hline o,p'-Methoxychlor & 19 & 12 & 5 & 7 & 6 & 8 & 4 & 2 & 3 & 3 & 3 \\
\hline p,p'-Methoxychlor & 17 & 17 & 13 & 10 & 9 & 16 & 6 & 5 & 8 & 5 & 7 \\
\hline cis-Chlordane & 12 & 13 & 8 & 9 & 4 & 11 & 4 & 3 & 6 & 3 & 6 \\
\hline trans-Chlordane & 11 & 10 & 7 & 7 & 5 & 11 & 5 & 5 & 5 & 1 & 2 \\
\hline Pentachlorobenzene & 19 & 17 & 13 & 13 & 9 & 20 & 7 & 5 & 8 & 6 & 7 \\
\hline Hexachlorobenzene & 19 & 17 & 13 & 13 & 9 & 20 & 7 & 5 & 8 & 6 & 7 \\
\hline o,p'-DDD & 19 & 17 & 13 & 13 & 9 & 20 & 6 & 5 & 8 & 6 & 7 \\
\hline p,p'-DDD & 18 & 17 & 13 & 13 & 9 & 20 & 7 & 4 & 8 & 6 & 7 \\
\hline$o, p^{\prime}-\mathrm{DDE}$ & 0 & 3 & 0 & 0 & 0 & 1 & 1 & 0 & 2 & 0 & 1 \\
\hline $\mathrm{p}, \mathrm{p}^{\prime}-\mathrm{DDE}$ & 14 & 17 & 10 & 10 & 5 & 12 & 4 & 2 & 7 & 4 & 5 \\
\hline o,p'-DDT & 19 & 12 & 3 & 10 & 6 & 15 & 4 & 4 & 5 & 3 & 5 \\
\hline p,p'-DDT & 19 & 13 & 13 & 12 & 9 & 15 & 6 & 5 & 7 & 6 & 5 \\
\hline $\begin{array}{l}\alpha \text {-Hexachloro } \\
\text { cyclohexane }\end{array}$ & 18 & 17 & 13 & 13 & 9 & 19 & 7 & 4 & 8 & 6 & 6 \\
\hline $\begin{array}{l}\beta \text {-Hexachloro } \\
\text { cyclohexane }\end{array}$ & 19 & 17 & 13 & 13 & 9 & 20 & 5 & 4 & 8 & 6 & 6 \\
\hline $\begin{array}{l}\gamma \text {-Hexachloro } \\
\text { cyclohexane }\end{array}$ & 19 & 17 & 13 & 13 & 9 & 20 & 6 & 4 & 8 & 5 & 6 \\
\hline $\begin{array}{c}\delta \text {-Hexachloro } \\
\text { cyclohexane }\end{array}$ & 19 & 17 & 13 & 13 & 8 & 17 & 7 & 5 & 8 & 6 & 6 \\
\hline
\end{tabular}

S - sesame oil, O - olive oil, C - corn oil, Co - colza oil, Ca - camellia oil, P - peanut oil, So - soybean, L - linseed, B - blend oil, Su - sunflower oil, R - rice oil. 
Table S3. Spearman's Rank Correlation Coefficient between Concentrations of OCPs in vegetable oils

\begin{tabular}{|c|c|c|c|c|c|c|c|c|c|c|c|c|c|}
\hline & A & B & $\mathrm{C}$ & $\mathrm{D}$ & E & $\mathrm{F}$ & $\mathrm{G}$ & $\mathrm{H}$ & I & $\mathrm{J}$ & $\mathrm{K}$ & $\mathrm{L}$ & M \\
\hline A & 1 & 0.127 & 0.025 & 0.186 & 0.764 & 0.183 & 0.125 & 0.220 & 0.017 & 0.028 & 0.138 & 0.330 & 0.021 \\
\hline B & & 1 & 0.042 & 0.109 & 0.033 & 0.086 & 0.028 & 0.087 & 0.478 & 0.003 & 0.133 & -0.087 & 0.096 \\
\hline $\mathrm{C}$ & & & 1 & -0.062 & 0.054 & 0.140 & -0.124 & 0.060 & 0.227 & 0.045 & 0.133 & 0.290 & -0.008 \\
\hline $\mathrm{D}$ & & & & 1 & 0.157 & 0.005 & 0.172 & 0.243 & -0.062 & 0.109 & -0.071 & -0.013 & -0.040 \\
\hline $\mathrm{E}$ & & & & & 1 & 0.120 & 0.108 & 0.226 & 0.130 & 0.161 & 0.269 & 0.393 & 0.062 \\
\hline $\mathrm{F}$ & & & & & & 1 & -0.210 & -0.127 & 0.331 & 0.030 & 0.161 & 0.360 & 0.234 \\
\hline G & & & & & & & 1 & 0.478 & -0.141 & 0.308 & 0.073 & -0.022 & -0.069 \\
\hline $\mathrm{H}$ & & & & & & & & 1 & 0.094 & 0.206 & 0.090 & 0.031 & 0.040 \\
\hline I & & & & & & & & & 1 & 0.167 & 0.283 & 0.332 & 0.559 \\
\hline $\mathrm{J}$ & & & & & & & & & & 1 & 0.042 & 0.088 & 0.096 \\
\hline K & & & & & & & & & & & 1 & 0.484 & 0.222 \\
\hline $\mathrm{L}$ & & & & & & & & & & & & 1 & 0.236 \\
\hline M & & & & & & & & & & & & & 1 \\
\hline
\end{tabular}

A - chlorobenzenes, B - HCHs, C - chlordene, D - heptachlor, E - aldrin, F - isodrin, G - 1-hydroxychlordene, H - chlordanes, I - DDTs, J - dieldrin, $\mathrm{K}$ - endrins, L - methoxychlors, $\mathrm{M}$ - mirex. 
Table S4. DI for the pesticides in different vegetable oils for urban and rural households (ng/kg bw/day)

\begin{tabular}{|c|c|c|c|c|c|c|c|c|c|c|c|c|c|c|c|c|c|c|c|c|c|c|}
\hline & \multicolumn{2}{|c|}{1} & \multicolumn{2}{|c|}{2} & \multicolumn{2}{|c|}{3} & \multicolumn{2}{|c|}{4} & \multicolumn{2}{|c|}{5} & \multicolumn{2}{|c|}{6} & \multicolumn{2}{|c|}{7} & \multicolumn{2}{|c|}{8} & \multicolumn{2}{|c|}{9} & \multicolumn{2}{|c|}{10} & \multicolumn{2}{|c|}{11} \\
\hline & $\mathrm{U}$ & $\mathrm{R}$ & $\mathrm{U}$ & $\mathrm{R}$ & $\mathrm{U}$ & $\mathrm{R}$ & $\mathrm{U}$ & $\mathrm{R}$ & $\mathrm{U}$ & $\mathrm{R}$ & $\mathrm{U}$ & $\mathrm{R}$ & $\mathrm{U}$ & $\mathrm{R}$ & $\mathrm{U}$ & $\mathrm{R}$ & $\mathrm{U}$ & $\mathrm{R}$ & $\mathrm{U}$ & $\mathrm{R}$ & $\mathrm{U}$ & $\mathrm{R}$ \\
\hline $\mathrm{a}$ & 0.93 & 0.83 & 1.25 & 1.12 & 0.22 & 0.19 & 0.04 & 0.03 & 0.63 & 0.56 & 0.10 & 0.09 & 0.40 & 0.36 & 0.47 & 0.42 & 0.79 & 0.71 & 1.57 & 1.40 & 0.06 & 0.06 \\
\hline b & 1.55 & 1.38 & 2.03 & 1.82 & 0.46 & 0.41 & 0.05 & 0.05 & 0.78 & 0.70 & 0.13 & 0.11 & 0.08 & 0.07 & 1.05 & 0.93 & 0.61 & 0.54 & 0.54 & 0.48 & 0.03 & 0.02 \\
\hline $\mathrm{c}$ & 1.01 & 0.90 & 1.41 & 1.26 & 0.15 & 0.14 & 0.06 & 0.05 & 0.52 & 0.46 & 0.13 & 0.12 & 0.05 & 0.05 & 0.23 & 0.20 & 0.50 & 0.45 & 0.18 & 0.16 & 0.02 & 0.02 \\
\hline d & 1.33 & 1.19 & 1.62 & 1.44 & 0.16 & 0.15 & 0.05 & 0.04 & 0.59 & 0.53 & 0.14 & 0.13 & 0.15 & 0.14 & 0.31 & 0.27 & 0.19 & 0.17 & 0.41 & 0.37 & 0.04 & 0.03 \\
\hline e & 1.28 & 1.15 & 1.74 & 1.56 & 0.22 & 0.19 & 0.06 & 0.05 & 0.60 & 0.54 & 0.13 & 0.12 & 0.04 & 0.04 & 0.51 & 0.46 & 0.32 & 0.29 & 0.45 & 0.40 & 0.02 & 0.02 \\
\hline $\mathrm{f}$ & 0.84 & 0.75 & 1.32 & 1.18 & 0.08 & 0.07 & 0.04 & 0.04 & 0.38 & 0.34 & 0.11 & 0.10 & 0.05 & 0.04 & 0.24 & 0.21 & 0.47 & 0.42 & 0.18 & 0.16 & 0.02 & 0.02 \\
\hline $\mathrm{g}$ & 1.72 & 1.54 & 2.12 & 1.90 & 0.28 & 0.25 & 0.05 & 0.04 & 0.74 & 0.66 & 0.18 & 0.16 & 0.09 & 0.08 & 0.21 & 0.19 & 0.69 & 0.61 & 0.48 & 0.43 & 0.02 & 0.02 \\
\hline $\mathrm{h}$ & 1.38 & 1.23 & 1.94 & 1.73 & 0.14 & 0.13 & 0.06 & 0.05 & 0.65 & 0.58 & 0.08 & 0.08 & 0.30 & 0.27 & 1.04 & 0.93 & 0.72 & 0.65 & 1.33 & 1.19 & 0.02 & 0.02 \\
\hline $\mathrm{i}$ & 1.74 & 1.55 & 2.20 & 1.96 & 0.23 & 0.21 & 0.05 & 0.05 & 0.83 & 0.74 & 0.13 & 0.12 & 0.09 & 0.08 & 0.28 & 0.25 & 0.52 & 0.47 & 0.99 & 0.89 & 0.02 & 0.02 \\
\hline $\mathrm{j}$ & 0.94 & 0.84 & 1.31 & 1.17 & 0.12 & 0.11 & 0.04 & 0.04 & 0.51 & 0.46 & 0.06 & 0.06 & 0.03 & 0.03 & 0.18 & 0.16 & 0.38 & 0.34 & 0.24 & 0.22 & 0.01 & 0.01 \\
\hline $\mathrm{k}$ & 1.48 & 1.32 & 2.08 & 1.85 & 0.14 & 0.13 & 0.03 & 0.03 & 0.67 & 0.60 & 0.11 & 0.10 & 0.03 & 0.03 & 0.07 & 0.06 & 0.60 & 0.54 & 0.80 & 0.72 & 0.02 & 0.02 \\
\hline
\end{tabular}

1-pentachlorobenzene, 2-hexachlorobenzene, 3- $\delta$-Hexachlorocyclohexane, 4-Heptachlor, 5-aldrin, 6-chlordane, 7-p,p'-DDT, 8-dieldrin, 9-endrin, 10-methoxychlor,

11-mirex. a - sesame oil, b - olive oil, c - corn oil, d - colza oil, e - camellia oil, f - peanut oil, g - soybean oil, h - linseed oil, i - blend oil, j - sunflower seed oil, k - rice oil. U - urban households, R - rural households. 
Table S5. HQ for the pesticides in different vegetable oils for urban and rural households

\begin{tabular}{|c|c|c|c|c|c|c|c|c|c|c|c|c|c|c|c|c|c|c|c|c|c|c|}
\hline & \multicolumn{2}{|c|}{1} & \multicolumn{2}{|c|}{2} & \multicolumn{2}{|c|}{3} & \multicolumn{2}{|c|}{4} & \multicolumn{2}{|c|}{5} & \multicolumn{2}{|c|}{6} & \multicolumn{2}{|c|}{7} & \multicolumn{2}{|c|}{8} & \multicolumn{2}{|c|}{9} & \multicolumn{2}{|c|}{10} & \multicolumn{2}{|c|}{11} \\
\hline & $\mathrm{U}$ & $\mathrm{R}$ & $\mathrm{U}$ & $\mathrm{R}$ & $\mathrm{U}$ & $\mathrm{R}$ & $\mathrm{U}$ & $\mathrm{R}$ & $\mathrm{U}$ & $\mathrm{R}$ & $\mathrm{U}$ & $\mathrm{R}$ & $\mathrm{U}$ & $\mathrm{R}$ & $\mathrm{U}$ & $\mathrm{R}$ & $\mathrm{U}$ & $\mathrm{R}$ & $\mathrm{U}$ & $\mathrm{R}$ & $\mathrm{U}$ & $\mathrm{R}$ \\
\hline \multirow{2}{*}{ a } & 1.16 & 1.04 & 1.57 & 1.40 & 7.24 & 6.47 & 7.75 & 6.92 & 2.10 & 1.88 & 2.09 & 1.87 & 8.07 & 7.21 & 9.31 & 8.32 & 2.64 & 2.36 & 3.14 & 2.81 & 3.13 & 2.80 \\
\hline & E-03 & E-03 & E-03 & E-03 & E-04 & E-04 & E-05 & E-05 & E-02 & E-02 & E-04 & E-04 & E-04 & E-04 & E-03 & E-03 & E-03 & E-03 & E-04 & E-04 & E-04 & E-04 \\
\hline \multirow{2}{*}{ b } & 1.93 & 1.73 & 2.54 & 2.27 & 1.55 & 1.38 & 1.05 & 9.37 & 2.60 & 2.32 & 2.57 & 2.29 & 1.60 & 1.43 & 2.09 & 1.87 & 2.02 & 1.81 & 1.08 & 9.62 & 1.31 & 1.17 \\
\hline & E-03 & E-03 & E-03 & E-03 & E-03 & E-03 & E-04 & E-05 & E-02 & E-02 & E-04 & E-04 & E-04 & E-04 & E-02 & E-02 & E-03 & E-03 & E-04 & E-05 & E-04 & E-04 \\
\hline \multirow{2}{*}{ c } & 1.26 & 1.12 & 1.77 & 1.58 & 5.12 & 4.57 & 1.14 & 1.02 & 1.73 & 1.54 & 2.64 & 2.36 & 1.05 & 9.37 & 4.52 & 4.04 & 1.68 & 1.50 & 3.66 & 3.26 & 1.04 & 9.32 \\
\hline & E-03 & E-03 & E-03 & E-03 & E-04 & E-04 & E-04 & E-04 & E-02 & E-02 & E-04 & E-04 & E-04 & E-05 & E-03 & E-03 & E-03 & E-03 & E-05 & E-05 & E-04 & E-05 \\
\hline \multirow{2}{*}{ d } & 1.67 & 1.49 & 2.02 & 1.80 & 5.42 & 4.85 & 9.74 & 8.70 & 1.96 & 1.75 & 2.84 & 2.53 & 3.06 & 2.73 & 6.16 & 5.50 & 6.21 & 5.55 & 8.20 & 7.32 & 1.80 & 1.60 \\
\hline & E-03 & E-03 & E-03 & E-03 & E-04 & E-04 & E-05 & E-05 & E-02 & E-02 & E-04 & E-04 & E-04 & E-04 & E-03 & E-03 & E-04 & E-04 & E-05 & E-05 & E-04 & E-04 \\
\hline \multirow{2}{*}{ e } & 1.61 & 1.43 & 2.18 & 1.94 & 7.25 & 6.47 & 1.13 & 1.01 & 2.01 & 1.79 & 2.68 & 2.39 & 8.72 & 7.79 & 1.03 & 9.20 & 1.08 & 9.64 & 8.96 & 8.01 & 1.11 & 9.93 \\
\hline & E-03 & E-03 & E-03 & E-03 & E-04 & E-04 & E-04 & E-04 & E-02 & E-02 & E-04 & E-04 & E-05 & E-05 & E-02 & E-03 & E-03 & E-04 & E-05 & E-05 & E-04 & E-05 \\
\hline \multirow{2}{*}{ f } & 1.05 & 9.33 & 1.66 & 1.48 & 2.69 & 2.40 & 8.04 & 7.18 & 1.26 & 1.13 & 2.25 & 2.01 & 9.91 & 8.86 & 4.73 & 4.22 & 1.57 & 1.41 & 3.50 & 3.13 & 1.24 & 1.11 \\
\hline & E-03 & E-04 & E-03 & E-03 & E-04 & E-04 & E-05 & E-05 & E-02 & E-02 & E-04 & E-04 & E-05 & E-05 & E-03 & E-03 & E-03 & E-03 & E-05 & E-05 & E-04 & E-04 \\
\hline \multirow{2}{*}{$\mathrm{g}$} & 2.15 & 1.92 & 2.65 & 2.37 & 9.43 & 8.42 & 9.11 & 8.14 & 2.48 & 2.21 & 3.62 & 3.23 & 1.87 & 1.67 & 4.20 & 3.75 & 2.28 & 2.04 & 9.68 & 8.65 & 9.01 & 8.05 \\
\hline & E-03 & E-03 & E-03 & E-03 & E-04 & E-04 & E-05 & E-05 & E-02 & E-02 & E-04 & E-04 & E-04 & E-04 & E-03 & E-03 & E-03 & E-03 & E-05 & E-05 & E-05 & E-05 \\
\hline \multirow{2}{*}{ h } & 1.73 & 1.54 & 2.42 & 2.16 & 4.72 & 4.22 & 1.13 & 1.01 & 2.17 & 1.94 & 1.68 & 1.50 & 6.05 & 5.40 & 2.08 & 1.86 & 2.42 & 2.16 & 2.66 & 2.38 & 1.23 & 1.10 \\
\hline & E-03 & E-03 & E-03 & E-03 & E-04 & E-04 & E-04 & E-04 & E-02 & E-02 & E-04 & E-04 & E-04 & E-04 & E-02 & E-02 & E-03 & E-03 & E-04 & E-04 & E-04 & E-04 \\
\hline \multirow{2}{*}{$\mathrm{i}$} & 2.17 & 1.94 & 2.75 & 2.45 & 7.83 & 6.99 & 1.09 & 9.77 & 2.75 & 2.46 & 2.59 & 2.31 & 1.70 & 1.52 & 5.66 & 5.06 & 1.75 & 1.56 & 1.99 & 1.78 & 1.16 & 1.04 \\
\hline & E-03 & E-03 & E-03 & E-03 & E-04 & E-04 & E-04 & E-05 & E-02 & E-02 & E-04 & E-04 & E-04 & E-04 & E-03 & E-03 & E-03 & E-03 & E-04 & E-04 & E-04 & E-04 \\
\hline \multirow{2}{*}{$\mathrm{j}$} & 1.17 & 1.05 & 1.64 & 1.46 & 4.04 & 3.61 & 8.92 & 7.97 & 1.70 & 1.52 & 1.25 & 1.11 & 6.63 & 5.92 & 3.62 & 3.23 & 1.26 & 1.13 & 4.89 & 4.37 & 7.01 & 6.26 \\
\hline & E-03 & E-03 & E-03 & E-03 & E-04 & E-04 & E-05 & E-05 & E-02 & E-02 & E-04 & E-04 & E-05 & E-05 & E-03 & E-03 & E-03 & E-03 & E-05 & E-05 & E-05 & E-05 \\
\hline \multirow[b]{2}{*}{ 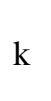 } & 1.85 & 1.65 & 2.60 & 2.32 & 4.68 & 4.18 & 6.27 & 5.60 & 2.25 & 2.01 & 2.16 & 1.93 & 6.08 & 5.43 & 1.35 & 1.21 & 2.00 & 1.79 & 1.61 & 1.43 & 1.04 & 9.31 \\
\hline & E-03 & E-03 & E-03 & E-03 & E-04 & E-04 & E-05 & E-05 & E-02 & E-02 & E-04 & E-04 & E-05 & E-05 & E-03 & E-03 & E-03 & E-03 & E-04 & E-04 & E-04 & E-05 \\
\hline
\end{tabular}


1-pentachlorobenzene, 2-hexachlorobenzene, 3- $\delta$-Hexachlorocyclohexane, 4-Heptachlor, 5-aldrin, 6-chlordane, 7-p,p'-DDT, 8-dieldrin, 9-endrin, 10-methoxychlor, 11-mirex. a - sesame oil, b - olive oil, c - corn oil, d - colza oil, e - camellia oil, f - peanut oil, g - soybean oil, h - linseed oil, i - blend oil, j - sunflower seed oil, k - rice oil. U - urban households, R - rural households. 